\title{
Molecular regulation of HDL metabolism and function: implications for novel therapies
}

\author{
Daniel J. Rader
}

Institute for Translational Medicine and Therapeutics, Cardiovascular Institute, and Institute for Diabetes, Obesity, and Metabolism, University of Pennsylvania School of Medicine, Philadelphia, Pennsylvania, USA.

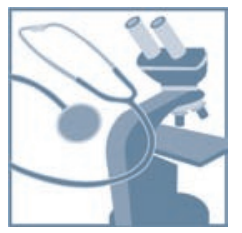

\begin{abstract}
HDL metabolism represents a major target for the development of therapies intended to reduce the risk of atherosclerotic cardiovascular disease. HDL metabolism is complex and involves dissociation of HDL apolipoprotein and HDL cholesterol metabolism. Advances in our understanding of the molecular regulation of HDL metabolism, macrophage cholesterol efflux, and HDL function will lead to a variety of novel therapeutics.
\end{abstract}

\section{Historical perspective}

HDLs were first characterized by using ultracentrifugation of plasma to separate lipoproteins of different densities. Several studies were published in the 1970s reporting an inverse association between plasma HDL cholesterol (HDL-C) level and coronary heart disease (1), confirmed since then by numerous additional studies throughout the world. HDL-C was first endorsed as a formal independent risk factor for coronary heart disease in the 1980s and has since evolved into one of the few "traditional" risk factors used by clinicians to assess cardiovascular risk. The guidelines (2) define a low HDL-C level as less than $40 \mathrm{mg} / \mathrm{dl}$ and also identify low HDL-C as one of the core components of the metabolic syndrome.

HDL-C levels vary widely over more than an order of magnitude in humans. This variation is at least $50 \%$, and as much as $70 \%$, genetically determined. Only a handful of genes have been proven to contribute to the genetic variation of HDL-C in humans, and they do so primarily by influencing the rate of HDL catabolism. Furthermore, the condition most commonly associated with low HDL-C, the insulin-resistant metabolic syndrome, is also associated with increased catabolism of HDL (3). Thus, HDL catabolism is a target for development of new therapies to raise HDL.

An important debate over the years has been whether low HDL-C is simply a marker of cardiovascular risk or is causally associated with increased risk. While low HDL-C is often seen in association with other conditions that also increase cardiovascular risk, it is widely believed, based in large part on animal studies, that HDL can directly inhibit atherogenesis. The most popular mechanistic explanation has been that HDL facilitates uptake of peripheral cholesterol and its return to the liver for excretion in the bile and feces, a concept first introduced by Glomset in 1968 (4) and subsequently termed "reverse cholesterol transport" (RCT). Later Ross and Glomset suggested that RCT could be a protective mechanism

Nonstandard abbreviations used: CE, cholesteryl ester; CETP, CE transfer protein; CVD, cardiovascular disease; EL, endothelial lipase; HDL-C, HDL cholesterol; HL, hepatic lipase; LCAT, lecithin:cholesterol acyltransferase; LDLR, LDL receptor; LXR, liver X receptor; PL, phospholipid; PLTP, PL transfer protein; RCT, reverse cholesterol transport; SR-BI, scavenger receptor class BI; TG, triglyceride.

Conflict of interest: The author has received research support, consulting fees, or honoraria from Abbott, AstraZeneca, Boehringer Ingelheim, Bristol-Myers Squibb, Bruin Pharma, GlaxoSmithKline, Johnson \& Johnson, KOS Pharmaceuticals Inc., Merck, Merck/Schering-Plough, Pfizer, Reliant Pharmaceuticals, Sanofi-Aventis, Schering-Plough, Takeda Pharmaceuticals North America, and Wyeth.

Citation for this article: J. Clin. Invest. 116:3090-3100 (2006). doi:10.1172/JCI30163. against atherosclerosis (5), and Miller and Miller suggested that HDL might protect against atherosclerosis by promoting RCT (1). While the ability of HDL to protect against atherosclerosis by promoting RCT mostly remains a hypothesis, promotion of RCT is nonetheless a primary target for the development of novel therapeutics targeted toward HDL. More recently, a variety of other functions of HDL have been described, primarily based on in vitro assays, including antiinflammatory, antioxidant, antithrombotic, and nitric oxide-inducing mechanisms $(6,7)$. Their relevance to human physiology remains uncertain. However, these findings have raised the important question of whether a substantial portion of the "protective" effect of HDL may be due to functions beyond RCT and whether these functions of HDL can be enhanced without increasing plasma levels of HDL-C per se.

It remains to be definitively proven that raising HDL-C or improving its function will reduce the risk of cardiovascular events. Results of some clinical trials have demonstrated that treating patients who have low HDL-C with therapies that raise HDL-C can reduce major coronary events (2). However, new therapeutic approaches to raising HDL-C levels and/or improving HDL function are required to conclusively prove this hypothesis. This review focuses on recent discoveries and remaining questions regarding the molecular regulation of HDL metabolism and function (including RCT) and the status of novel therapeutics being developed based on these discoveries.

\section{HDL biosynthesis}

Apolipoprotein production. The biosynthesis of HDL (Figure 1) is complex and involves the synthesis and secretion of the major protein components of HDL followed by the largely extracellular acquisition of lipid (phospholipids [PLs] and cholesterol) and the assembly and generation of the mature HDL particle. The major HDL apolipoproteins are apoA-I and apoA-II, and both are required for normal HDL biosynthesis. ApoA-I constitutes approximately $70 \%$ of HDL protein and is present on virtually all HDL particles (3). Thus, it is not surprising that gene deletion of Apoa1/ $A P O A 1$ results in extremely low levels of HDL-C in mice (8) and in humans (9). Atherosclerosis-prone mice lacking apoA-I develop significantly increased atherosclerosis (10).

ApoA-I is synthesized in both the intestine and the liver (Figure 1), but the relative contribution of the intestine and the liver to the plasma apoA-I pool in humans remains unknown. Hepatic overex- 

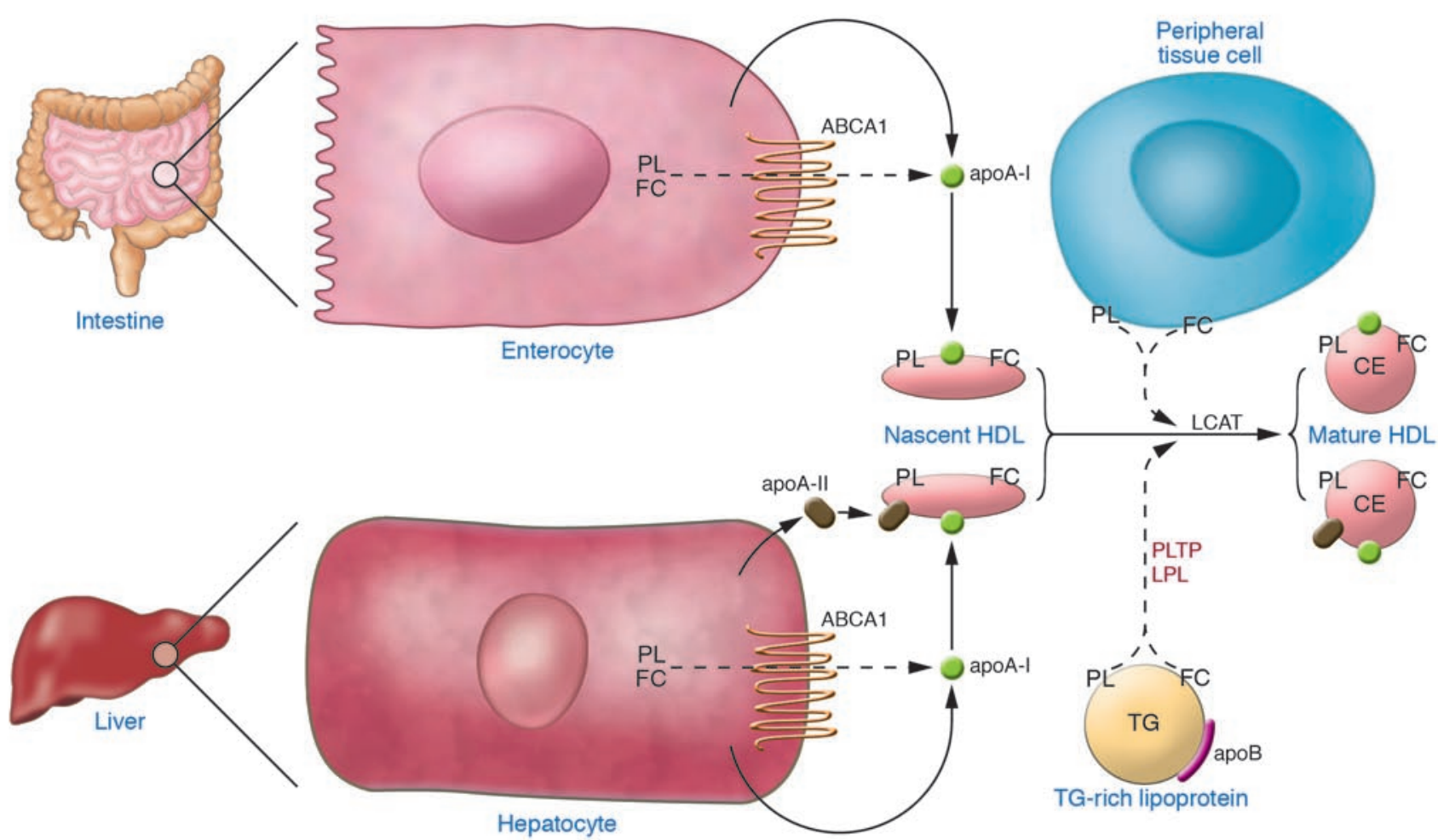

\section{Figure 1}

HDL biosynthesis. Enterocytes and hepatocytes synthesize apoA-I, which is secreted in a lipid-poor form and then immediately recruits additional PLs and free cholesterol (FC) via the ABCA1 pathway, forming nascent HDL. Nascent HDL acquires more lipid from other peripheral tissues and from lipoproteins, and LCAT generates CE, forming mature HDL. The liver also synthesizes apoA-II, which results in a subclass of HDL containing both apoA-I and apoA-II.

pression of apoA-I significantly raises HDL-C levels and inhibits the progression of and even regresses atherosclerosis in mice (11-13). Thus, upregulation of endogenous apoA-I expression is widely considered one of the most promising approaches to the development of new therapies targeted to HDL. Much has been learned about the regulation of apoA-I transcription (14). PPAR $\alpha$ agonists, such as fibrates, have been shown to upregulate transcription of the human $A P O A 1$ gene in human $A P O A 1$ transgenic mice in vivo (15). However, fibrates are relatively weak PPAR $\alpha$ agonists, have modest effects on apoA-I concentrations in humans, and have not been conclusively demonstrated to increase apoA-I production in humans; more potent PPAR $\alpha$ agonists could potentially have a greater effect. The orphan nuclear receptor liver receptor homolog-1 (LRH-1) was shown to upregulate apoA-I transcription through direct binding to the apoA-I promoter (16), whereas the transcriptional repressor small heterodimer partner suppresses apoA-I transcription by inhibiting the activity of LRH-1. While knowledge regarding the transcriptional regulation of apoA-I has not yet produced small molecules that have advanced in clinical development, this remains an area of active interest.

ApoA-II constitutes approximately $20 \%$ of HDL protein, is present on about two-thirds of HDL particles in humans, and is synthesized only in the liver. Gene deletion of Apoa2 in mice markedly reduces HDL-C levels (17), suggesting that apoA-II is also required for normal HDL biosynthesis and metabolism. While overexpression of apoA-II in mice raises levels of HDL-C, it increases atherosclerosis $(18,19)$. In both mice and humans, quantitative trait loci linkage studies suggest that the Apoa2/APOA2 gene locus is a determinant of HDL-C levels (20). Unlike apoA-I, plasma apoA-II levels are determined primarily by the rate of production (21). Interestingly, fibrates (PPAR $\alpha$ agonists), thiazolidinediones (PPAR $\gamma$ agonists), and alcohol all significantly increase plasma apoA-II levels in concert with increased HDL-C levels. Thus, increasing APOA2 gene transcription could be a strategy for raising HDL-C levels; however, the implications of this strategy for atherosclerosis in humans are uncertain.

Lipid acquisition and maturation. Newly secreted HDL apolipoproteins must acquire lipid (PLs and cholesterol) in order to generate HDL particles. Recent studies have established that the lipidation of HDL apolipoproteins occurs primarily after their secretion and that ABCA 1 is a critical participant in the early lipidation of newly secreted apoA-I. Genetic absence of ABCA1 forms the molecular basis of Tangier disease (22-24), which is associated with extremely low levels of HDL-C and apoA-I. Abca1-knockout mice have a phenotype similar to that of Tangier disease patients (25). The production rate of apoA-I in Tangier disease is normal, but apoA-I is extremely rapidly catabolized (26). Although it is ubiquitously expressed, ABCA1 in the liver and the intestine appears to be responsible for the vast majority of the initial lipidation of lipidpoor apoA-I (Figure 1). Mice that lack ABCA1 specifically in the liver have HDL-C levels that are reduced by $80 \%$ (27), and mice that lack ABCA1 in the intestine have a $30 \%$ reduction in HDL-C (28). Upregulation of hepatic and/or intestinal ABCA1 could potentially be a therapeutic strategy for raising HDL-C levels. 


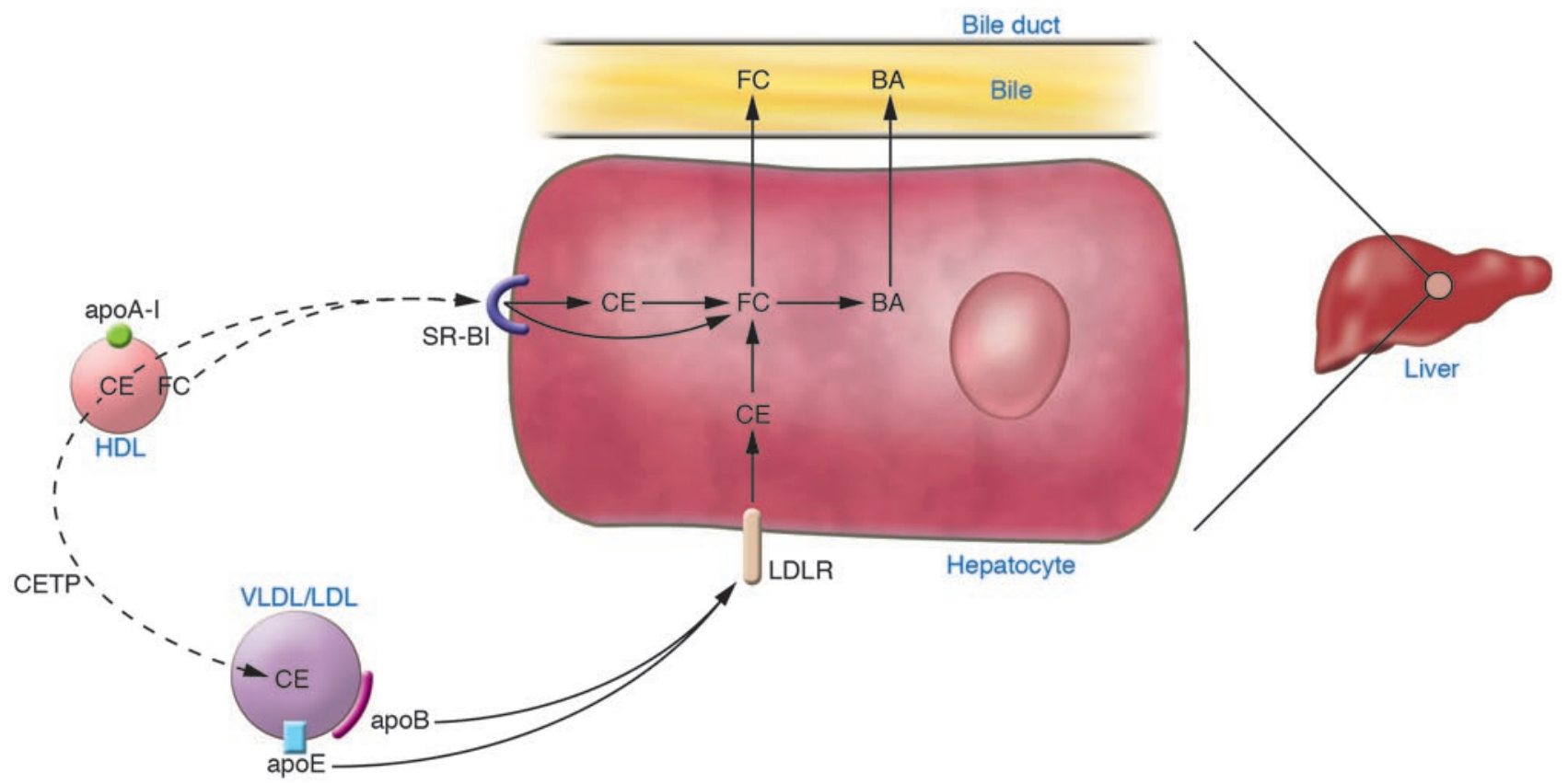

Figure 2

Pathways of HDL cholesterol uptake by the liver. HDL-CE and HDL free cholesterol can be directly and selectively taken up by the liver via SR-BI. Alternatively, HDL-CE can be transferred to apoB-containing lipoproteins by CETP and then taken up by liver via the LDLR. In the hepatocyte, CE is hydrolyzed to free cholesterol, which is either excreted directly into the bile or converted to bile acid (BA) and excreted into the bile.

While the liver and intestine are critical for initial lipidation of apoA-I via ABCA1, HDL derives much of its lipid mass from other sources. The potential additional sources include other tissues and other lipoproteins (Figure 1). In mice, about $90 \mathrm{mg}$ cholesterol per kilogram of body weight is effluxed daily from extrahepatic tissues to HDL (29). Very little is known about the sources or mechanisms of extrahepatic lipidation of HDL. Macrophages, the most important cell type to efflux cholesterol from the point of view of atherosclerosis, almost certainly do not contribute much cholesterol mass to HDL. For example, when wild-type bone marrow was transplanted into ABCA1-knockout mice, there was little increase in plasma HDL-C levels (30). Because all extrahepatic cells require cholesterol and yet cannot metabolize it, they need to efflux cholesterol to HDL. Thus, it stands to reason that the most important contributors to the HDL cholesterol pool outside the liver and intestine would be large organ beds such as skeletal muscle, adipose tissue, and skin. Indeed, skeletal myocytes, adipocytes, and skin fibroblasts have all been shown to have the ability to efflux cholesterol to acceptors such as apoA-I and HDL in vitro. More research is needed to determine the relative contribution of these and other organs to the lipidation of HDL and the pathways and molecular mechanisms by which efflux of PLs and cholesterol from these tissues occurs. This issue is not purely academic, as pharmacologic upregulation of efflux pathways of lipids from extrahepatic tissues to HDL could result in increased levels of HDL-C.

HDL also derives lipids, particularly PLs, from other lipoproteins (Figure 1). When triglyceride-rich (TG-rich) lipoproteins undergo hydrolysis of the TG core, surface PLs (and apolipoproteins) are shed and acquired by HDL. Thus, the activity of lipoprotein lipase is inversely associated with HDL-C levels (3). Lipoprotein-derived PLs are transferred to HDL by the PL transfer protein (PLTP) (31).
Mice lacking PLTP have a significant reduction in HDL-C levels (32), and mice overexpressing PLTP have increased levels of HDL-C (33). The role of PLTP in humans, and whether PLTP is a target for therapeutic development, remain to be determined.

The development of mature HDL requires the esterification of cholesterol to form cholesteryl ester (CE) and the hydrophic lipid core of HDL. HDL-CE is formed by the action of lecithin:cholesterol acyltransferase (LCAT), an HDL-associated enzyme that catalyzes the transfer of a fatty acid from PL to free cholesterol (Figure 1). LCAT is critical for the maintenance of normal HDL metabolism. LCAT deficiency in humans (34) and in mice (35) causes markedly reduced levels of HDL-C and rapid catabolism of apoA-I and apoA-II (36). Conversely, overexpression of LCAT in mice results in substantially increased HDL-C levels (37). LCAT has been considered to be important in the process of RCT by generating a gradient of free cholesterol from cells to HDL, but the impact of LCAT activity on RCT in vivo has not been examined. In humans, free cholesterol in HDL can be directly transferred to the liver and secreted in bile (38). Nevertheless, upregulation of LCAT activity would be expected to increase HDL-C levels and therefore is a potential goal for the development of new therapies.

\section{HDL catabolism}

HDL cholesterol catabolism. A detailed understanding of the pathways for HDL cholesterol catabolism is extremely important for development of novel therapeutics. The flux of cholesterol through these HDL pathways may be more important than the steady-state HDL-C level. The major site of HDL cholesterol uptake is the liver (Figure 2). The best-understood mechanism of direct uptake of HDL cholesterol by the liver is that mediated by the scavenger receptor class BI (SR-BI). Importantly, SR-BI promotes hepatic 
uptake of HDL cholesterol (both esterified and unesterified) without mediating degradation of HDL apolipoproteins, a process known as selective uptake (Figure 2). Studies of SR-BI in polarized hepatocytes suggest that SR-BI mediates internalization of whole particles of HDL, with subsequent removal of cholesterol and resecretion of smaller cholesterol-depleted HDL particles (39). Abundant data indicate that in rodents SR-BI is a critical regulator of HDL metabolism. Hepatic overexpression of SR-BI in mice markedly increases hepatic HDL cholesterol uptake and reduces plasma HDL-C levels (40). Conversely, deletion of the SR-BI gene Scarb1 in mice slows hepatic HDL cholesterol uptake and increases plasma HDL-C levels $(41,42)$. In mice, hepatic SR-BI expression appears to be an important overall regulator of RCT: SR-BI overexpression increased RCT, and SR-BI deficiency reduced RCT (43). This is consistent with the observations that hepatic SR-BI overexpression reduced atherosclerosis despite reducing HDL-C levels (44-46) and that Scarb1-knockout mice have markedly increased atherosclerosis despite increased HDL-C levels (47-49).

Overall, this body of data is probably the best example of the concept that flux of HDL cholesterol is more important than steady-state concentrations with regard to atherosclerosis. These observations have led to the concept that upregulation of hepatic SR-BI could be antiatherogenic despite the reduced plasma HDL-C. However, there remain questions regarding the physiologic importance of the hepatic SR-BI pathway for uptake of HDL cholesterol in humans. Studies in healthy, normolipidemic humans have suggested that relatively little HDL-CE is directly taken up by the liver and targeted to bile (38). Studies of SCARB1 genetic polymorphisms in humans have indicated only a modest association with variation in plasma HDL-C levels (50), and no SR-BI-deficient patients have been definitively reported to date. Based on results of murine studies, one might predict that the phenotype of SR-BI deficiency in humans is elevated HDL-C levels accompanied by increased risk of atherosclerotic cardiovascular disease (CVD).

In humans, there is clearly at least one alternative pathway by which HDL cholesterol is metabolized and ultimately transported to the liver, a pathway mediated by the CE transfer protein (CETP). CETP transfers TG from apoB-containing lipoproteins in exchange for HDL-CE, thus resulting in CE depletion and TG enrichment of HDL (Figure 2). Rodents naturally lack CETP, and when engineered to express it, they experience substantial reduction in HDL-C levels (51). The proof that CETP is important for human HDL metabolism came from the discovery of humans genetically deficient in CETP $(52,53)$. These individuals, who have loss-of-function mutations in both alleles of the CETP gene and are found almost exclusively in Japan, have extremely high levels of HDL-C. In addition, their HDL is exceptionally large, and the turnover of apoA-I is substantially slowed (54). Confirmation that the CETP pathway is quantitatively important for hepatic uptake of HDL-CE in humans was obtained in a study in which injection of HDL labeled with a CE tracer demonstrated that the labeled cholesterol that was excreted into bile was first largely transferred to apoB-containing lipoproteins (38).

The discovery of CETP deficiency raised the question of whether pharmacologic inhibition of CETP might be a novel approach to raise plasma HDL-C levels. CETP inhibitors are now the most advanced class of novel HDL level-raising drugs $(55,56)$. The CETP inhibitor JTT-705 was shown to raise HDL-C levels as monotherapy (57) and in combination with pravastatin (58). Another CETP inhibitor, torcetrapib, increased HDL-C levels in healthy volunteers (59) and in subjects with low baseline HDL-C levels (60). In subsequent phase II studies, dose-dependent increases in HDL-C levels were seen with torcetrapib alone as well as in combination with atorvastatin (61). Modest LDL-C lowering has also been reported with CETP inhibition that was proportional to the increase in HDL-C (58-61). Finally, in contrast to the small-molecule approach, a strategy of generating an immune response to CETP using a CETP-based peptide for immunization is in clinical development (62).

Whether CETP inhibition reduces CVD remains an open question. The relationship of CETP deficiency to CVD is uncertain: there are a relatively small number of CETP-deficient homozygotes, and population-based attempts to definitively address this question in homozygotes have not been carried out. Individuals heterozygous for CETP mutations have only a modest increase in HDL-C levels and have no major difference in cardiovascular risk (63). Additional genetic studies of CETP polymorphisms in other populations (64) and measurement of CETP in large prospective observational studies (65) are conflicting but suggest that increased CETP may be a CVD risk factor in humans. There is a possibility that CETP inhibition may not promote and could actually impair RCT, given the apparent importance of the CETP pathway in transporting HDL-CE to the liver (38). Administration of torcetrapib resulted in significantly slower catabolism of apoA-I but had no significant effect on fecal neutral sterol excretion (66). While an early report suggested that HDL from CETP-deficient subjects was "dysfunctional" in its ability to promote macrophage cholesterol efflux (67), results of a recent study indicate that CETP-deficient HDL is more effective than control HDL in promoting macrophage cholesterol efflux via the ABCG1 pathway (see below) (68). Determining the effects of CETP inhibition on atherosclerosis is the most direct method of providing insight into the effects of this strategy. Studies of CETP inhibition in rabbits have consistently shown a reduction in atherosclerosis (56). Atherosclerosis imaging trials with torcetrapib using intravascular ultrasound of the coronaries and measuring intimamedia thickness of the carotids, as well as a large cardiovascular outcome trial with torcetrapib, are in progress (69). The question of whether CETP inhibition reduces CVD is a critically important issue for the field of HDL therapeutics.

$H D L$ apoA-I catabolism. Results of kinetic studies in humans have demonstrated that the turnover rate of apoA-I is much more variable than its production rate and is a more important determinant of plasma apoA-I and HDL-C concentrations (3). Thus, understanding the sites and mechanisms of apoA-I catabolism is important (Figure 3). Studies in animals using trapped ligands (70) established that a substantial portion (approximately onethird) of apoA-I is catabolized by the kidneys, and the rest (greater than half) is catabolized by the liver. Note that the sites of apoA-I catabolism in humans are assumed to be similar but have never been formally studied.

More is understood regarding the mechanisms of apoA-I catabolism by the kidney than by the liver. Lipid-poor apoA-I can be filtered at the level of the glomerulus and then catabolized by proximal renal tubular epithelial cells (Figure 3). Cubilin is an extracellular protein synthesized by proximal renal tubular cells and localized to the apical surface anchored by another protein called "amnionless" (71). Cubilin binds HDL and apoA-I with high affinity $(72,73)$ and then interacts with a coreceptor called megalin, a member of the low-density lipoprotein receptor $(L D L R)$ gene family, to mediate uptake 


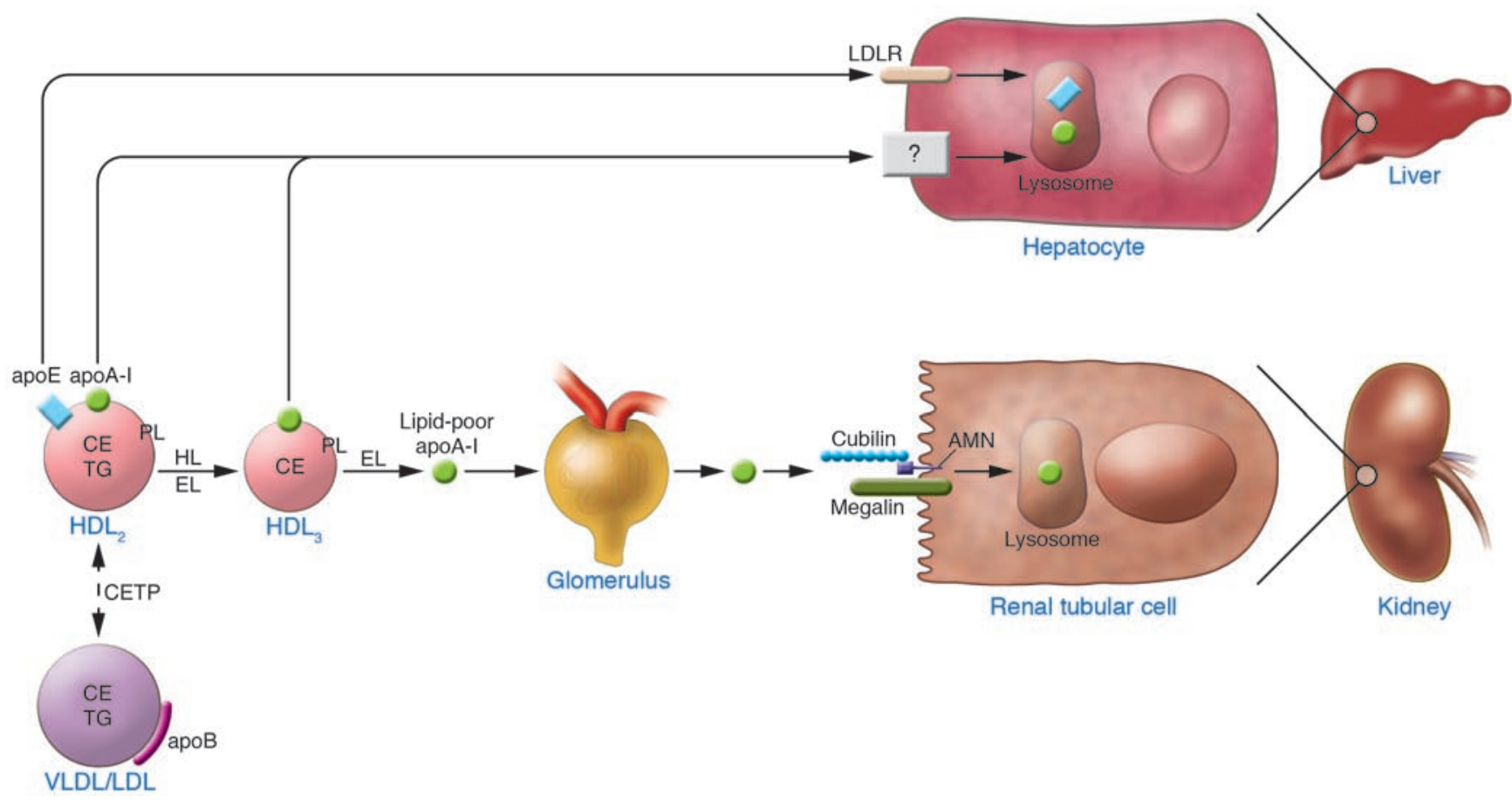

Figure 3

Pathways of apoA-I catabolism. Lipid-poor apoA-I can be filtered through the renal glomerulus and be degraded by the renal tubular cell via the cubulin-megalin pathway. Lipid-poor apoA-I is generated by the action of lipases such as $\mathrm{HL}$ and EL on mature HDL, and the action of these lipases is enhanced by the CETP-mediated transfer of TG into HDL. Mature HDL can also be catabolized by the hepatocyte through binding of HDL-apoE to hepatic receptors or through a poorly characterized mechanism of direct interaction with the hepatocyte. AMN, amnionless.

and degradation of apoA-I (74). However, functional cubilin deficiency in animals or humans is not associated with changes in plasma HDL-C or apoA-I concentrations (75). Thus, it is doubtful that this renal tubular pathway is responsible for variation in HDL-C levels in humans or is a target for the development of therapeutic approaches to raise HDL.

The rate-limiting step in the renal catabolism of apoA-I is probably at the glomerular filtration level rather than at the level of tubular catabolism. Therefore, there is major interest in the factors that regulate the rate of glomerular filtration of apoA-I. Mature $\mathrm{HDL}$ is too large to be filtered, and probably only apoA-I that is relatively lipid poor can be filtered to any extent. The extent of apoA-I lipidation thus plays a major role in the rate of renal apoA-I catabolism. ApoA-I lipidation is determined by both acquisition and maturation of lipid as well as by remodeling of the mature HDL particle. There are several examples from human pathophysiology of impaired lipid acquisition by apoA-I leading to increased apoA-I catabolism. As noted above, the best example is that of Tangier disease, in which the inability of the liver and the intestine to lipidate newly synthesized apoA-I via the ABCA1 pathway results in a poorly lipidated apoA-I that is rapidly catabolized (26) primarily by the kidneys (27). Importantly, even $A B C A 1$ heterozygotes have increased apoA-I catabolism (26), demonstrating a gene dose effect of $A B C A 1$ with regard to apoA-I turnover. Furthermore, in LCAT deficiency the lack of LCAT-mediated cholesterol esterification results in accelerated apoA-I catabolism (36), presumably because the formation of the CE core in HDL helps to generate particles of large enough size to escape renal glomerular filtration. However, heterozygotes for LCAT mutations have normal plasma
apoA-I levels and thus presumably normal apoA-I turnover, suggesting that LCAT is not a determinant of apoA-I turnover across a range of LCAT activity.

Mature HDL is "remodeled" by several factors, resulting in the generation of smaller HDL particles and lipid-poor apoA-I that is subject to glomerular filtration and catabolism. HDL remodeling consists of removal of CE as well as hydrolysis of PLs and TGs; these processes are often linked. The 2 most important processes involving removal of HDL-CE are transfer to other lipoproteins via CETP and selective uptake via SR-BI. Both are discussed in detail above, but it is worth noting here that both influence the turnover of apoA-I. For example, CETP deficiency in humans is associated with reduced apoA-I turnover (54), as is pharmacologic inhibition of CETP (66). Furthermore, overexpression of CETP (76) and SR-BI (77) in mice increases the turnover of apoA-I. Thus, factors that influence the amount of CE in HDL modulate the rate of apoA-I catabolism.

The modification of HDL by lipolytic enzymes is another important determinant of apoA-I turnover. The best-studied enzyme with regard to HDL metabolism is hepatic lipase (HL). HL has the ability to hydrolyze both TG and PL in HDL (Figure 3). A series of studies have demonstrated that HL is most effective in hydrolyzing HDL if the HDL is TG enriched (3), a process that occurs with increased CETP-mediated exchange of HDL-CE for TG from TGrich lipoproteins. The action of HL on TG-enriched HDL results in the shedding of lipid-poor apoA-I from HDL and increased apoA-I catabolism. Indeed, apoA-I associated with TG-enriched HDL is catabolized significantly faster than non-TG-enriched HDL (78). Insulin-resistant states, which are known to be associ- 
ated with increased rates of apoA-I catabolism, are also associated with increased HL activity (3). Thus, inhibition of HL would be expected to reduce HDL remodeling, slow apoA-I catabolism, and increase apoA-I and HDL-C levels. However, because HL may play a role in the clearance of apoB-containing remnant particles, inhibition of HL for therapeutic purposes is not so straightforward.

More recently, endothelial lipase (EL), which is closely related to $\mathrm{HL}$, has been described and characterized $(79,80)$. In contrast to HL, EL appears to have relatively more phospholipase activity than TG lipase activity and appears to have a greater preference for HDL over apoB-containing lipoproteins. Overexpression of EL in mice causes a reduction in HDL-C levels $(79,81)$ and also reduces apoA-I levels because of increased catabolism primarily via the kidneys (82). Conversely, antibody inhibition (83) or gene deletion $(81,84)$ of EL results in increased HDL-C and apoA-I levels. Subjects with high HDL-C levels may be more likely to have potentially functional mutations in the EL gene LIPG (85), but association studies of common polymorphisms in EL have not yet told a compelling story. Plasma EL levels in humans were found to be significantly inversely associated with HDL-C levels and positively associated with a variety of measures of the metabolic syndrome (86), which suggests that EL contributes to the low HDL-C levels that occur with insulin resistance. While results of atherosclerosis studies in EL knockout mice have been conflicting $(87,88)$, plasma EL levels in humans were found to be significantly associated with coronary atherosclerosis (86). Inhibition of EL is an attractive therapeutic approach that might be expected to reduce apoA-I catabolism and increase plasma apoA-I and HDL-C levels.

In animals, the liver is responsible for substantial degradation of apoA-I (70). The mechanisms of hepatic uptake and HDL apolipoprotein degradation remain poorly understood. One mechanism that likely plays at least some role is related to apoE. A portion of HDL particles contain apoE, and apoE-rich HDL is known to be a ligand for the LDL receptor (LDLR) and other apoE receptors (89). Thus this pathway may contribute to the uptake of some HDL by the liver (Figure 3). However, HDL depleted of apoE still has the ability to be taken up and degraded by hepatocytes, and other mechanisms must also exist. There has been substantial effort invested in identifying other hepatic HDL-binding proteins (90), but none have been proven to be bona fide HDL receptors in vivo. The $\beta$ chain of ATP synthase was reported to be ectopically localized to the hepatocyte plasma membrane, where it may act as a high-affinity receptor for HDL apoA-I to trigger the endocytosis of HDL particles in an ADP-dependent process (91). The nucleotide G protein-coupled receptor $\mathrm{P} 2 \mathrm{Y} 13$ was suggested to act as a participant in this process (92). Gain- and loss-of-function studies in animals and human genetic studies are required to assess the real physiologic role of this pathway and of other hepatic HDL-binding proteins.

A thorough understanding of the pathways and molecular regulation of hepatic apoA-I catabolism is critically important to the development of new therapeutic approaches. In concept, if the hepatic catabolism of apoA-I could be slowed without affecting the rate of HDL cholesterol turnover or RCT, this could be a novel approach to raising apoA-I concentrations and thus inhibiting atherosclerosis. In fact, the most effective pharmacologic method for raising HDL currently available is nicotinic acid (niacin), and studies in humans have suggested that niacin administration reduces the catabolic rate of apoA-I (93). While the molecular mechanism underlying this observation remains unknown, niacin has been shown to reduce uptake of HDL apoA-I in hepatocytes in vitro (94). It is possible that niacin acts on a hepatic "HDL receptor" or on an as-yet-unknown pathway to slow HDL apoA-I catabolism. A G protein-coupled receptor called GPR109A has been described as a niacin receptor (95), but is not thought to be highly expressed by hepatocytes. Instead, it is primarily expressed by adipocytes and its activation results in reduced release of fatty acids from adipose (94), which may explain niacin's effects on plasma TGs but does not obviously explain its effects on HDL. The molecular mechanisms by which niacin raises HDL-C levels have yet to be established. Nevertheless, niacin remains a useful example of how reducing hepatic HDL apoA-I catabolism might be a viable strategy for increasing HDL-C and apoA-I levels. Whether compounds specifically targeted toward activating GPR109A will have effects in reducing hepatic apoA-I catabolism, or any HDL-C raising properties at all, is a critically important question for the field. Further investigation of the pathways of hepatic catabolism of apoA-I could yield new targets for drug discovery.

\section{HDL function}

The above discussion focused on the molecular mechanisms that regulate plasma concentrations of HDL-C and apoA-I. These mechanisms have therapeutic implications, because increasing their production or reducing their catabolism has the potential to increase levels of HDL-C and apoA-I, and plasma levels are the most direct biomarker of an effect on HDL. The simplest paradigm for new therapeutic development would be one in which raising plasma HDL-C/apoA-I concentrations by any mechanism reliably translated into reduced atherosclerotic vascular disease, similar to that of reducing plasma LDL-C concentrations. However, this cannot be assumed: in theory, certain methods of raising HDL level could impair its function and not translate into vascular benefit (e.g., SR-BI deficiency in mice). Thus, for the foreseeable future any new therapy that raises HDL-C levels will need to demonstrate actual cardiovascular benefit, either through imaging atherosclerosis and/or through reduction in hard cardiovascular events. Nevertheless, the development of a new therapeutic approach that actually raises HDL-C levels provides a useful early biomarker and also raises hope that the increase in HDL-C will be beneficial.

On the other hand, it is also relatively easy to imagine the possibility that certain approaches could improve the function of $\mathrm{HDL}$ without necessarily increasing plasma HDL-C concentrations. For example, flux through the RCT pathway could theoretically be increased without increasing the steady-state level of HDL-C. The rate of RCT cannot be inferred from the plasma HDL-C level, but rather requires sophisticated kinetic measures of cholesterol flux (96). In this section, the molecular regulation of the functions of HDL that may contribute to its antiatherogenic effects and subsequent insights into the development of novel therapeutic approaches are reviewed.

Promoting macrophage cholesterol efflux and RCT. There has been a major emphasis over the last 2 decades in attempting to understand the molecular mechanisms by which the efflux of cholesterol from macrophages to HDL is regulated. Macrophages are professional phagocytes that take up dead and dying cells as well as aggregated and modified lipoproteins, all of which contain abundant cholesterol. Because too much cholesterol can be toxic, they have evolved several pathways to efflux cholesterol to the extracellular environment (Figure 4). The most-studied pathway for macrophage cholesterol efflux is the pathway by which the ABCA1 transporter promotes cholesterol efflux to lipid-poor apoA-I as an acceptor. Macrophages 


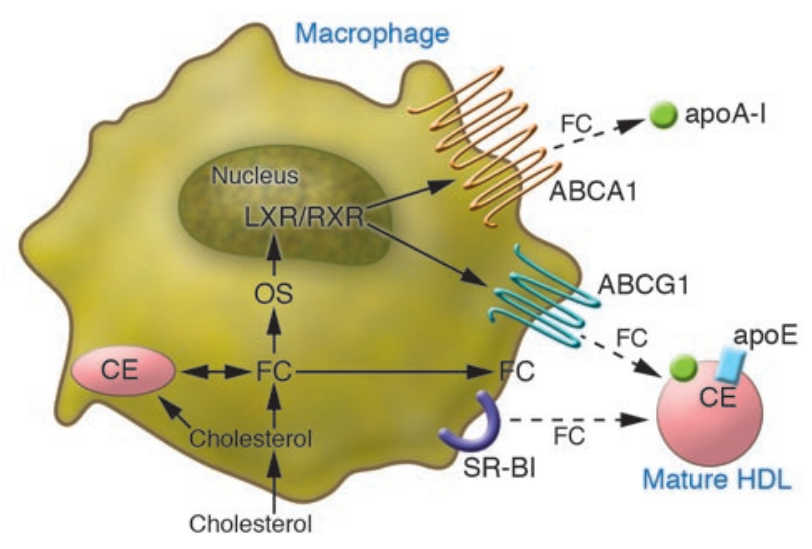

Figure 4

Pathways of cholesterol efflux from macrophages. Macrophages have several pathways for efflux of cholesterol. They efflux free cholesterol to lipid-poor apoA-I via the ABCA1 pathway and to mature HDL via the $A B C G 1$ pathway. Both $A B C A 1$ and $A B C G 1$ are regulated by the nuclear receptor $L X R$, which is activated by binding of oxysterols, derived from free cholesterol. SR-BI also has the capacity to efflux free cholesterol to mature HDL.

from ABCA1-knockout mice have substantially reduced cholesterol efflux to lipid-poor apoA-I as an acceptor (30). Indeed, transplantation of bone marrow from ABCA1-knockout mice into atherosclerosis-prone mice resulted in significantly increased atherosclerosis even though plasma HDL-C levels were virtually unchanged (30). Conversely, overexpression of ABCA1 in cells of mice, including but not limited to macrophages, was associated with a significant reduction in atherosclerosis (97). Thus, it is possible that upregulation of ABCA1 in macrophages could be a powerful antiatherogenic strategy. The major regulators of ABCA1 gene expression are the nuclear receptor liver X receptors $\alpha$ and $\beta$ (LXR $\alpha$ and LXR $\beta)$, which act as heterodimers with their partner the retinoid X receptor (98). LXRs are activated endogenously by oxysterols, which are generated enzymatically from cholesterol. Both oxysterols and synthetic LXR agonists upregulate $\mathrm{ABCA} 1$ transcription in macrophages and cause increased cholesterol efflux to lipid-poor apoA-I.

However, in vivo most of apoA-I is not lipid poor (the preferred acceptor for ABCA1) but in large, mature HDL particles. In fact, mature HDL is also capable of promoting cholesterol efflux from macrophages. It has recently been established that another member of the ABC transporter family, ABCG1, is capable of promoting cholesterol efflux from macrophages to mature HDL $(99,100)$ by an unknown mechanism. In macrophages, ABCG1 is transcriptionally upregulated as well as translocated to the plasma membrane by cholesterol loading or LXR agonists (101). ABCG1deficient macrophages have impaired cholesterol efflux to mature HDL, and ABCG1-knockout mice accumulate cholesterol and TGs in macrophages, particularly in the lung (100). The importance of ABCG1 in cholesterol efflux from macrophages in vivo remains uncertain. Somewhat paradoxically, macrophage deficiency of ABCG1 was shown to result in reduced atherosclerosis (102, 103), possibly due to compensatory upregulation of macrophage ABCA1 and apoE (102) or increased susceptibility of ABCG1-deficient macrophages to oxidized LDL-induced apoptosis (103).

Treatment of macrophages with LXR agonists results in upregulation of both $\mathrm{ABCA} 1$ and $\mathrm{ABCG} 1$ and increased cholester- ol efflux to both lipid-poor apoA-I and mature HDL. Furthermore, a synthetic LXR agonist was shown to significantly promote macrophage cholesterol efflux and RCT in vivo despite having little effect on plasma HDL-C levels (104). Synthetic LXR agonists have also been shown to inhibit the progression $(105,106)$, and even promote the regression (107), of atherosclerosis in mice despite having little effect on plasma HDL-C levels. Thus, administration of LXR agonists are a good example of the concept that RCT can be substantially promoted (and atherosclerosis inhibited) without actually raising plasma levels of HDL-C. As a result, LXR agonists have been viewed as an attractive novel therapeutic approach for atherosclerosis. However, some nonselective LXR agonists have been found to cause hepatic steatosis and hypertriglyceridemia in animals, which is believed to be due to induction of hepatic expression of SREBP1c and fatty acid synthetic genes (108). Furthermore, some LXR agonists increased LDL-C levels in animals that express CETP (109). Nevertheless, LXR remains a prime therapeutic target, and in theory more selective LXR modulators may be found that have the beneficial effects in macrophages without the adverse effects in the liver.

Alternative approaches to upregulating macrophage cholesterol efflux are being actively explored. Both PPAR $\alpha$ and PPAR $\gamma$ agonists have been shown to upregulate $A B C A 1$, promote macrophage cholesterol efflux in vitro $(110,111)$, and reduce the formation of foam cells in vivo (112). It has been suggested that PPAR $\alpha$ agonists may upregulate LXRs in macrophages $(110,111)$, and a PPAR $\alpha$ agonist was shown to inhibit foam cell formation in an LXRdependent (but ABCA1-independent) manner (112). It is interesting to consider that fibrates, relatively weak PPAR $\alpha$ agonists, may reduce cardiovascular risk in part by promoting macrophage cholesterol efflux and that more potent PPAR $\alpha$ agonists could be even more effective. It has also been suggested that PPAR $\gamma$ agonists may upregulate LXRs in macrophages, but a PPAR $\gamma$ agonist inhibited foam cell formation in an LXR-independent manner (112). $\operatorname{PPAR} \beta / \delta$ agonists have been suggested to promote macrophage cholesterol efflux (113), but this has not been definitively established. Studies are needed to formally test whether agonists of any of the PPARs are capable of promoting macrophage RCT in vivo.

It may be possible to functionally increase macrophage ABCA1 and ABCG1 through approaches other than transcriptional regulation. In addition, there remains substantial interest in identifying other pathways by which macrophages efflux cholesterol. For example, SR-BI is expressed in macrophages and can promote cholesterol efflux to mature HDL (114). It is uncertain whether macrophage SR-BI quantitatively participates in macrophage cholesterol efflux and under what conditions. However, bone marrow transplantation from SR-BI-deficient mice into LDLR-deficient (49) or apoE-deficient (115) mice resulted in increased atherosclerosis, consistent with an atheroprotective role of macrophage SR-BI. There may be other regulated efflux pathways in the macrophage as well. It is anticipated that therapies that promote macrophage cholesterol efflux by upregulating one or more of the efflux pathways will eventually be developed for clinical use.

Because efflux is in part dependent on the concentration of the acceptor, it is also logical to attempt to increase the concentration of the acceptor. As noted above, increasing endogenous production of apoA-I might be considered the ideal approach. However, exogenous approaches have also been explored; particular attention has been given to lipid-poor apoA-I in this regard. Intravenous bolus infusion of apoA-I in humans results in a substantial, 
but transient, increase in lipid-poor apoA-I (116). A single bolus of pro-apoA-I did not raise HDL-C levels but resulted in a significant increase in fecal sterol excretion, suggesting promotion of RCT (117). Infusion of apoA-I $\mathrm{I}_{\text {Milano/phosphotidylcholine complexes }}$ weekly for 5 weeks was associated with modest regression of coronary atheroma volume from baseline as measured by intravascular ultrasound (118), although these results must be interpreted with caution (119). Selective delipidation of HDL ex vivo, which generates lipid-poor apoA-I, might be able to be reinfused in an autologous fashion (120). Finally, the concept of using apoA-I mimetic peptides rather than full-length apoA-I is being actively explored (121). Amphipathic peptides of 18-22 amino acids have been developed that have properties similar to those of apoA-I, including the ability to promote cholesterol efflux, activate LCAT, and reduce inflammation. Repeated injection of the peptide L-5F reduced the progression of atherosclerosis in mice (122). Oral administration of related peptide D-4F also reduced progression of atherosclerosis (123). D-4F was shown to promote macrophage cholesterol efflux in vitro and RCT in vivo (124) and is in early clinical development. The apoA-I mimetic peptide ETC-642 (also known as RLT peptide) promotes LCAT activation and is also in clinical development. Several other apoA-I mimetic peptides have been developed and tested in cell and animal models (121). ApoA-I mimetic peptides may ultimately prove to be an effective method of promoting macrophage cholesterol efflux, improving other aspects of HDL function (see below), and inhibiting atherosclerosis in humans.

Promoting antiinflammatory and other beneficial effects of $H D L$. In addition to cholesterol efflux promotion, several additional, potentially antiatherogenic properties of HDL have been described. These include inhibition of LDL oxidation, inhibition of endothelial inflammation, promotion of endothelial nitric oxide production, promotion of prostacyclin bioavailability, and inhibition of platelet aggregation and coagulation $(6,7)$. The molecular mechanisms of these effects have yet to be fully elucidated, and the importance of these mechanisms to the atheroprotective effects of HDL remains uncertain. However, in concept they are potentially quite important. First, strategies that raise the level of HDL through inhibiting its catabolism may be antiatherogenic even if they do not promote cholesterol efflux and RCT by working through these other mechanisms. Second, therapies may be developed that enhance these functions of HDL even without increasing HDL-C levels per se. Third, once the molecular mechanisms are better understood, therapies may be developed that directly mimic these HDL effects on the vasculature.

Perhaps the best current example of an approach that may increase HDL function is the apoA-I mimetic peptide D-4F mentioned above. D-4F is composed of all $\mathrm{D}$ amino acids and therefore is not recognized by gut peptidases and is partially absorbed after oral dosing. Some data suggest that D-4F enhances the antiinflammatory function of HDL in mice without increasing HDL-C levels (121), although the mechanism of this effect is uncertain and this finding must be confirmed in humans. D-4F may permit the first test of the hypothesis that enhancing HDL function without increasing plasma HDL-C levels can reduce atherosclerosis or cardiovascular risk.

\section{Future directions}

Aggressive reduction of LDL-C is a cornerstone of preventive cardiovascular care, but additional therapeutic approaches to reduce atherosclerosis progression (or induce its regression) and prevent cardiovascular events are still needed. HDL is an obvious and attractive target, but current therapeutic approaches to targeting HDL are inadequate. There are relatively few validated targets for developing novel therapeutic approaches targeted toward HDL. The most advanced, CETP inhibition, is in late-stage clinical development, and the outcome may have a tremendous impact on the field. If CETP inhibitors are shown to be effective in reducing atherosclerotic CVD and approved, they will likely become widely used clinically. In this setting, the potential for additional HDLtargeted therapies will need to be carefully assessed. The probability is that additional HDL-based therapies will be complementary to CETP inhibition and find their role, much as additional LDLlowering approaches beyond statins are still needed for many patients. For example, it may still be very useful to upregulate macrophage efflux pathways (i.e., LXR agonists) in order to more effectively promote cholesterol efflux to the increased HDL acceptors induced by inhibition of CETP, or it may be possible to further improve the function of the HDL (i.e., apoA-I mimetic peptides) in the setting of CETP inhibition.

On the other hand, if CETP inhibitors are ineffective in reducing atherosclerosis, it will not mean that the overall concept of targeting HDL for therapeutic purposes is flawed. As discussed above, CETP inhibition is complex because it raises HDL-C levels by fundamentally slowing the metabolism of HDL cholesterol. In the event that CETP inhibitors are found to be ineffective, the need to develop other HDL-based therapeutics that work through different mechanisms will be even more imperative. While some of the current targets for small molecule development such as LXR, $\mathrm{EL}$, and the niacin receptor have potential, there is still a dearth of validated targets for HDL therapeutics. Additional efforts are needed to better understand the molecular regulation of HDL metabolism as well as to identify additional genes contributing to variation of HDL-C levels in humans. Indeed, some of the most important insights regarding molecular targets for HDL-based therapies have been derived from studies of human genetic syndromes (e.g., Tangier disease and CETP deficiency). Genome-wide studies in mice and humans (20) promise to yield new, previously unsuspected genes involved in HDL metabolism, some of which will become targets for new therapeutic development.

The concept that acute parenteral administration of a synthetic form of HDL to patients presenting with acute coronary syndromes may reduce the high short-term risk of recurrent events is attractive and plausible. Results of animal studies indicate that regression and changes in plaque morphology consistent with "stabilization" can occur rapidly after the introduction of an HDLbased therapeutic, and at least one clinical trial is consistent with this notion. Multiple approaches to the concept are being taken, and one or more of these approaches may ultimately prove to effectively reduce cardiovascular events after acute coronary syndromes and be introduced to the market. Acute induction therapy for unstable coronary disease would then become standard in inpatient settings, with maintenance therapy in outpatient settings continuing for weeks or months after discharge. This would be a radical change in the paradigm of treating coronary disease.

For all novel HDL-based therapeutics, the challenge is determining early in development whether a particular strategy is likely to be effective with regard to atherosclerosis. Raising plasma HDL-C levels is neither adequate nor necessary for a successful HDL-targeted therapy. There remains tremendous need for new in vivo kinetic approaches and novel biomarkers to reliably assess the effects 
of new therapies on RCT and HDL function and predict their effects on atherosclerosis. Close collaboration between companies developing HDL-based therapeutics and academicians expert in the complex assessment of RCT and HDL function in vivo will be required to develop the necessary tools for early assessment of promising therapies. In summary, the efforts of the basic science community to understand the molecular basis of HDL metabolism and function have yielded important insights that are leading to new therapeutic advances. The next few decades will likely be to HDL-based intervention what the last couple of decades have been to LDL-based intervention: a time of definitive proof of the "HDL hypothesis" and an expansion of the therapeutic armamentarium for manipulating HDL in the interest of further reducing the risk and burden of atherosclerotic CVD.

\section{Acknowledgments}

The author has been supported by National Heart, Lung, and Blood Institute grants HL55323, HL22633, HL62250, HL59407, and HL70128; National Institute of Diabetes and Digestive and Kidney Diseases grants DK59533 and DK06990; National Center for Research Resources grant M01 RR00040; the Burroughs Wellcome Fund Clinical Scientist Award in Translational Research; and a Doris Duke Charitable Foundation Distinguished Clinical Scientist Award.

Address correspondence to: Daniel J. Rader, University of Pennsylvania Medical Center, 654 BRBII/III Labs, 421 Curie Boulevard, Philadelphia, Pennsylvania 19104-6160, USA. Phone: (215) 5734176; Fax: (215) 573-8606; E-mail: rader@mail.med.upenn.edu.
1. Miller, G.J., and Miller, N.E. 1975. Plasma-highdensity-lipoprotein concentration and development of ischaemic heart-disease. Lancet. 1:16-19.

2. Expert Panel on Detection, Evaluation, and Treatment of High Blood Cholesterol in Adults (Adult Treatment Panel III). 2001. Executive summary of the third report of the National Cholesterol Education Program (NCEP). JAMA. 285:2486-2497.

3. Lewis, G.F., and Rader, D.J. 2005. New insights into the regulation of HDL metabolism and reverse cholesterol transport. Circ. Res. 96:1221-1232.

4. Glomset, J.A. 1968. The plasma lecithins:cholesterol acyltransferase reaction. J. Lipid Res. 9:155-167.

5. Ross, R., and Glomset, J.A. 1973. Atherosclerosis and the arterial smooth muscle cell: proliferation of smooth muscle is a key event in the genesis of the lesions of atherosclerosis. Science. 180:1332-1339.

6. Barter, P.J., et al. 2004. Antiinflammatory properties of HDL. Circ. Res. 95:764-772.

7. Mineo, C., Deguchi, H., Griffin, J.H., and Shaul, P.W. 2006. Endothelial and antithrombotic actions of HDL. Circ. Res. 98:1352-1364.

8. Williamson, R., Lee, D., Hagaman, J., and Maeda, N. 1992. Marked reduction of high density lipoprotein cholesterol in mice genetically modified to lack apolipoprotein A-I. Proc. Natl. Acad. Sci. U. S. A 89:7134-7138.

9. Schaefer, E.J., Heaton, W.H., Wetzel, M.G., and Brewer, H.B., Jr. 1982. Plasma apolipoprotein A-1 absence associated with a marked reduction of high density lipoproteins and premature coronary artery disease. Arteriosclerosis. 2:16-26.

10. Moore, R.E., et al. 2005. Increased atherosclerosis in mice lacking apolipoprotein A-I attributable to both impaired reverse cholesterol transport and increased inflammation. Circ. Res. 97:763-771.

11. Rubin, E., Krauss, R., Spangler, E., Verstuyft, J., and Clift, S. 1991. Inhibition of early atherogenesis in transgenic mice by human apolipoprotein AI. Nature. 353:265-267.

12. Plump, A., Scott, C., and Breslow, J. 1994. Human apolipoprotein A-I gene expression increases high density lipoprotein and suppresses atherosclerosis in the apolipoprotein E-deficient mouse. Proc. Natl. Acad. Sci. U. S. A. 91:9607-9611.

13. Tangirala, R.K., et al. 1999. Regression of atherosclerosis induced by liver-directed gene transfer of apolipoprotein A-I in mice. Circulation. 100:1816-1822.

14. Mooradian, A.D., Haas, M.J., and Wong, N.C. 2004. Transcriptional control of apolipoprotein A-I gene expression in diabetes. Diabetes. 53:513-520.

15. Berthou, L., et al. 1996. Opposite regulation of human versus mouse apolipoprotein A-I by fibrates in human apolipoprotein A-I transgenic mice. J. Clin. Invest. 97:2408-2416.

16. Delerive, P., Galardi, C.M., Bisi, J.E., Nicodeme, E., and Goodwin, B. 2004. Identification of liver receptor homolog-1 as a novel regulator of apolipoprotein $\mathrm{AI}$ gene transcription. Mol. Endocrinol. 18:2378-2387.

17. Weng, W., and Breslow, J.L. 1996. Dramatically decreased high density lipoprotein cholesterol, increased remnant clearance, and insulin hypersensitivity in apolipoprotein A-II knockout mice suggest a complex role for apolipoprotein A-II in atherosclerosis susceptibility. Proc. Natl. Acad. Sci. U. S. A. 93:14788-14794.

18. Schultz, J.R., Verstuyft, J.G., Gong, E.L., Nichols, A.V., and Rubin, E.M. 1993. Protein composition determines the anti-atherogenic properties of HDL in transgenic mice. Nature. 365:762-764.

19. Warden, C.H., Hedrick, C.C., Qiao, J.-H., Castellani, L.W., and Lusis, A.J. 1993. Atherosclerosis in transgenic mice overexpressing apolipoprotein A-II. Science. 261:469-472.

20. Wang, X., and Paigen, B. 2005. Genetics of variation in HDL cholesterol in humans and mice. Circ. Res. 96:27-42.

21. Ikewaki, K., Zech, L.A., Kindt, M., Brewer, H.B., Jr., and Rader, D.J. 1995. Apolipoprotein A-II production rate is a major factor regulating the distribution of apolipoprotein A-I among HDL subclasses LPA-I and LPA-I:A-II in normolipidemic humans. Arterioscler.Thromb. Vasc. Biol. 15:306-312.

22. Rust, S., et al. 1999. Tangier disease is caused by mutations in the gene encoding ATP-binding cassette transporter 1. Nat. Genet. 22:352-355.

23. Bodzioch, M., et al. 1999. The gene encoding ATPbinding cassette transporter 1 is mutated in Tangier disease. Nat. Genet. 22:347-351.

24. Brooks-Wilson, A., et al. 1999. Mutations in ABC1 in Tangier disease and familial high-density lipoprotein deficiency. Nat. Genet. 22:336-345.

25. McNeish, J., et al. 2000. High density lipoprotein deficiency and foam cell accumulation in mice with targeted disruption of ATP-binding cassette transporter-1. Proc. Natl. Acad. Sci. U. S. A. 97:4245-4250.

26. Schaefer, E.J., et al. 1978. Metabolism of high-density lipoprotein apolipoproteins in Tangier disease. N. Engl.J. Med. 299:905-910.

27. Timmins, J.M., et al. 2005. Targeted inactivation of hepatic Abca1 causes profound hypoalphalipoproteinemia and kidney hypercatabolism of apoA-I. J. Clin. Invest. 115:1333-1342. doi:10.1172/ JCI200523915.

28. Brunham, L.R., et al. 2006. Intestinal ABCA1 directly contributes to HDL biogenesis in vivo. J. Clin. Invest. 116:1052-1062. doi:10.1172/JCI27352.

29. Dietschy, J.M., and Turley, S.D. 2002. Control of cholesterol turnover in the mouse. J. Biol. Chem. 277:3801-3804.

30. Haghpassand, M., Bourassa, P.A., Francone, O.L., and Aiello, R.J. 2001. Monocyte/macrophage expression of ABCA1 has minimal contribution to plasma HDL levels. J. Clin. Invest. 108:1315-1320. doi:10.1172/JCI200112810.

31. Huuskonen, J., Olkkonen, V.M., Jauhiainen, M., and Ehnholm, C. 2001. The impact of phospho- lipid transfer protein (PLTP) on HDL metabolism. Atherosclerosis. 155:269-281.

32. Jiang, X.C., et al. 1999. Targeted mutation of plasma phospholipid transfer protein gene markedly reduces high-density lipoprotein levels. J. Clin. Invest. 103:907-914.

33. Jiang, X., et al. 1996. Increased pre $\beta$-high density lipoprotein, apolipoprotein AI, and phospholipid in mice expressing the human phospholipid transfer protein and human apolipoprotein AI transgenes. J. Clin. Invest. 98:2373-2380.

34. Kuivenhoven, J.A., et al. 1997. The molecular pathology of lecithin:cholesterol acyltransferase (LCAT) deficiency syndromes. J. Lipid Res. 38:191-205.

35. $\mathrm{Ng}, \mathrm{D}$.S. 2004. Insight into the role of LCAT from mouse models. Rev. Endocr. Metab. Disord. 5:311-318.

36. Rader, D.J., et al. 1994. Markedly accelerated catabolism of apolipoprotein A-II (ApoA-II) and high density lipoproteins containing ApoA-II in classic lecithin: cholesterol acyltransferase deficiency and fish-eye disease. J. Clin. Invest. 93:321-330.

37. Francone, O.L., Gong, E.L., Ng, D.S., Fielding, C.J., and Rubin, E.M. 1995. Expression of human lecithin-cholesterol acyltransferase in transgenic mice. Effect of human apolipoprotein AI and human apolipoprotein all on plasma lipoprotein cholesterol metabolism. J. Clin. Invest. 96:1440-1448.

38. Schwartz, C.C., VandenBroek, J.M., and Cooper, P.S. 2004. Lipoprotein cholesteryl ester production, transfer, and output in vivo in humans. J. Lipid Res. 45:1594-1607.

39. Silver, D.L., Wang, N., Xiao, X., and Tall, A.R. 2001. High density lipoprotein (HDL) particle uptake mediated by scavenger receptor class B type 1 results in selective sorting of HDL cholesterol from protein and polarized cholesterol secretion. J. Biol. Chem. 276:25287-25293.

40. Kozarsky, K.F., et al. 1997. Overexpression of the HDL receptor SR-B1 alters plasma HDL and bile cholesterol levels. Nature. 387:414-417.

41. Rigotti, A., et al. 1997. A targeted mutation in the murine gene encoding the high density lipoprotein (HDL) receptor scavenger receptor class B type I reveals its key role in HDL metabolism. Proc. Natl. Acad. Sci. U. S. A. 94:12610-12615.

42. Brundert, M., et al. 2005. Scavenger receptor class B type I mediates the selective uptake of high-density lipoprotein-associated cholesteryl ester by the liver in mice. Arterioscler. Thromb. Vasc. Biol. 25:143-148.

43. Zhang, Y., et al. 2005. Hepatic expression of scavenger receptor class B type I (SR-BI) is a positive regulator of macrophage reverse cholesterol transport in vivo. J. Clin. Invest. 115:2870-2874. doi:10.1172/ JCI25327.

44. Kozarsky, K.F., Donahee, M.H., Glick, J.M., Krieger, M., and Rader, D.J. 2000. Gene transfer and hepatic overexpression of the HDL receptor SR-BI reduces atherosclerosis in the cholesterol-fed LDL receptor-deficient mouse. Arterioscler. Thromb. Vasc. Biol. 
20:721-727.

45. Arai, T., Wang, N., Bezouevski, M., Welch, C. and Tall, A.R. 1999. Decreased atherosclerosis in heterozygous low density lipoprotein receptordeficient mice expressing the scavenger receptor BI transgene. J. Biol. Chem. 274:2366-2371.

46. Ueda, Y., et al. 2000. Relationship between expression levels and atherogenesis in scavenger receptor class B, type I transgenics. J. Biol. Chem. 275:20368-20373.

47. Trigatti, B., et al. 1999. Influence of the high density lipoprotein receptor SR-BI on reproductive and cardiovascular pathophysiology. Proc. Natl. Acad. Sci.U. S. A. 96:9322-9327.

48. Braun, A., et al. 2002. Loss of SR-BI expression leads to the early onset of occlusive atherosclerotic coronary artery disease, spontaneous myocardial infarctions, severe cardiac dysfunction, and premature death in apolipoprotein E-deficient mice. Circ. Res. 90:270-276.

49. Covey, S.D., Krieger, M., Wang, W., Penman, M., and Trigatti, B.L. 2003. Scavenger receptor class B type I-mediated protection against atherosclerosis in LDL receptor-negative mice involves its expression in bone marrow-derived cells. Arterioscler. Thromb. Vasc. Biol. 23:1589-1594.

50. Osgood, D., et al. 2003. Genetic variation at the scavenger receptor class B type I gene locus determines plasma lipoprotein concentrations and particle size and interacts with type 2 diabetes: the framingham study. J. Clin. Endocrinol. Metab. 88:2869-2879.

51. Agellon, L.B., et al. 1991. Reduced high density lipoprotein cholesterol in human cholesteryl ester transfer protein transgenic mice. J. Biol. Chem. 266:10796-10801.

52. Brown, M.L., et al. 1989. Molecular basis of lipid transfer protein deficiency in a family with increased high-density lipoproteins. Nature. 342:448-451.

53. Inazu, A., et al. 1990. Increased high-density lipoprotein levels caused by a common cholesteryl-ester transfer protein gene mutation. N. Engl. J. Med. 323:1234-1238.

54. Ikewaki, K., et al. 1993. Delayed catabolism of high density lipoprotein apolipoproteins A-I and A-II in human cholesteryl ester transfer protein deficiency. J. Clin. Invest. 92:1650-1658.

55. De Grooth, G.J., et al. 2004. A review of CETP and its relation to atherosclerosis. J. Lipid Res. 45:1967-1974.

56. Rader, D.J. 2004. Inhibition of cholesteryl ester transfer protein activity: a new therapeutic approach to raising high-density lipoprotein. Curr. Atheroscler. Rep. 6:398-405.

57. De Grooth, G.J., et al. 2002. Efficacy and safety of a novel cholesteryl ester transfer protein inhibitor, JTT-705, in humans: a randomized phase II doseresponse study. Circulation. 105:2159-2165.

58. Kuivenhoven, J.A., et al. 2005. Effectiveness of inhibition of cholesteryl ester transfer protein by JTT-705 in combination with pravastatin in type II dyslipidemia. Am. J. Cardiol. 95:1085-1088.

59. Clark, R.W., et al. 2004. Raising high-density lipoprotein in humans through inhibition of cholesteryl ester transfer protein: an initial multidose study of torcetrapib. Arterioscler. Thromb. Vasc. Biol. 24:490-497.

60. Brousseau, M.E., et al. 2004. Effects of an inhibitor of cholesteryl ester transfer protein on HDL cholesterol. N. Engl. J. Med. 350:1505-1515.

61. Davidson, M.H., McKenney, J.M., Shear, C.L., and Revkin, J.H. 2006. Efficacy and safety of torcetrapib, a novel cholesteryl ester transfer protein inhibitor, in individuals with below-average high-density lipoprotein cholesterol levels. J. Am. Coll. Cardiol. 48:1774-1781.

62. Davidson, M.H., et al. 2003. The safety and immu- nogenicity of a CETP vaccine in healthy adults. Atherosclerosis. 169:113-120.

63. Curb, J.D., et al. 2004. A prospective study of HDL-C and cholesteryl ester transfer protein gene mutations and the risk of coronary heart disease in the elderly. J. Lipid Res. 45:948-953.

64. Boekholdt, S.M., and Thompson, J.F. 2003. Natural genetic variation as a tool in understanding the role of CETP in lipid levels and disease. J. Lipid Res. 44:1080-1093.

65. Boekholdt, S.M., et al. 2004. Plasma levels of cholesteryl ester transfer protein and the risk of future coronary artery disease in apparently healthy men and women: the prospective EPIC (European Prospective Investigation into Cancer and nutrition)-Norfolk population study. Circulation. 110:1418-1423.

66. Brousseau, M.E., et al. 2005. Effects of cholesteryl ester transfer protein inhibition on high-density lipoprotein subspecies, apolipoprotein A-I metabolism, and fecal sterol excretion. Arterioscler. Thromb. Vasc. Biol. 25:1057-1064.

67. Ishigami, M., et al. 1994. Large and cholesteryl ester-rich high-density lipoproteins in cholesteryl ester transfer protein (CETP) deficiency can not protect macrophages from cholesterol accumulation induced by acetylated low-density lipoproteins. J. Biochem. (Tokyo). 116:257-262.

68. Matsuura, F., Wang, N., Chen, W., Jiang, X.C., and Tall, A.R. 2006. HDL from CETP-deficient subjects shows enhanced ability to promote cholesterol efflux from macrophages in an apoE- and ABCG1dependent pathway. J. Clin. Invest. 116:1435-1442. doi:10.1172/JCI27602.

69. Bays, H., McKenney, J., and Davidson, M. 2005. Torcetrapib/atorvastatin combination therapy. Expert Rev. Cardiovasc. Ther. 3:789-820.

70. Glass, C., Pittman, R.C., Weinstein, D.B., and Steinberg, D. 1983. Dissociation of tissue uptake of cholesterol ester from that of apoprotein A-I of rat plasma high density lipoprotein: selective delivery of cholesterol ester to liver, adrenal, and gonad. Proc. Natl. Acad. Sci. U. S. A. 80:5435-5439.

71. Moestrup, S.K., and Nielsen, L.B. 2005. The role of the kidney in lipid metabolism. Curr. Opin. Lipidol. 16:301-306.

72. Hammad, S.M., et al. 1999. Cubilin, the endocytic receptor for intrinsic factor-vitamin B12 complex, mediates high-density lipoprotein holoparticle endocytosis. Proc. Natl. Acad. Sci. U. S. A. 96:10158-10163.

73. Kozyraki, R., et al. 1999. The intrinsic factorvitamin B12 receptor, cubilin, is a high-affinity apolipoprotein A-I receptor facilitating endocytosis of high-density lipoprotein. Nat. Med. 5:656-661.

74. Hammad, S.M., Barth, J.L., Knaak, C., and Argraves, W.S. 2000. Megalin acts in concert with cubilin to mediate endocytosis of high density lipoproteins. J. Biol. Chem. 275:12003-12008.

75. Christensen, E.I., and Gburek, J. 2004. Protein reabsorption in renal proximal tubule-function and dysfunction in kidney pathophysiology. Pediatr. Nephrol. 19:714-721.

76. Hayek, T., et al. 1992. An interaction between the human cholesteryl ester transfer protein (CETP) and apolipoprotein A-I genes in transgenic mice results in a profound CETP-mediated depression of high density lipoprotein cholesterol levels. J. Clin. Invest. 90:505-510.

77. Webb, N.R., et al. 2002. Overexpression of SR-BI by adenoviral vector promotes clearance of apoA-I, but not apoB, in human apoB transgenic mice. J. Lipid Res. 43:1421-1428.

78. Lamarche, B., et al. 1999. Triglyceride enrichment of HDL enhances in vivo metabolic clearance of HDL apo A-I in healthy men. J. Clin. Invest. 103:1191-1199.

79. Jaye, M., et al. 1999. A novel endothelial-derived lipase that modulates HDL metabolism. Nat. Genet.
21:424-428.

80. Hirata, K., et al. 1999. Cloning of a unique lipase from endothelial cells extends the lipase gene family. J. Biol. Chem. 274:14170-14175.

81. Ishida, T., et al. 2003. Endothelial lipase is a major determinant of HDL level. J. Clin. Invest. 111:347-355. doi:10.1172/JCI200316306.

82. Maugeais, C., et al. 2003. Dose-dependent acceleration of high-density lipoprotein catabolism by endothelial lipase. Circulation. 108:2121-2126.

83. Jin, W., Millar, J.S., Broedl, U., Glick, J.M., and Rader, D.J. 2003. Inhibition of endothelial lipase causes increased HDL cholesterol levels in vivo. J. Clin. Invest. 111:357-362. doi:10.1172/JCI200316146.

84. Ma, K., et al. 2003. Endothelial lipase is a major genetic determinant for high-density lipoprotein concentration, structure, and metabolism. Proc. Natl. Acad. Sci. U. S. A. 100:2748-2753.

85. deLemos, A.S., Wolfe, M.L., Long, C.J., Sivapackianathan, R., and Rader, D.J. 2002. Identification of genetic variants in endothelial lipase in persons with elevated high-density lipoprotein cholesterol. Circulation. 106:1321-1326.

86. Badellino, K.O., Wolfe, M.L., Reilly, M.P., and Rader, D.J. 2006. Endothelial lipase concentrations are increased in metabolic syndrome and associated with coronary atherosclerosis. PLoS Med. 3:e22.

87. Ishida, T., et al. 2004. Endothelial lipase modulates susceptibility to atherosclerosis in apolipoproteinE-deficient mice. J. Biol. Chem. 279:45085-45092.

88. Ko, K.W., Paul, A., Ma, K., Li, L., and Chan, L. 2005. Endothelial lipase modulates HDL but has no effect on atherosclerosis development in apoE-/and LDLR-/- mice. J. Lipid Res. 46:2586-2594.

89. Mahley, R.W., Huang, Y., and Weisgraber, K.H. 2006. Putting cholesterol in its place: apoE and reverse cholesterol transport. J. Clin. Invest. 116:1226-1229. doi:10.1172/JCI28632.

90. Fidge, N.H. 1999. High density lipoprotein receptors, binding proteins, and ligands. J. Lipid Res. 40:187-201.

91. Martinez, L.O., et al. 2003. Ectopic beta-chain of ATP synthase is an apolipoprotein A-I receptor in hepatic HDL endocytosis. Nature. 421:75-79.

92. Jacquet, S., et al. 2005. The nucleotide receptor $\mathrm{P} 2 \mathrm{Y} 13$ is a key regulator of hepatic high-density lipoprotein (HDL) endocytosis. Cell. Mol. Life Sci. 62:2508-2515.

93. Carlson, L.A. 2005. Nicotinic acid: the broad-spectrum lipid drug. A 50th anniversary review. J. Intern. Med. 258:94-114.

94. Meyers, C.D., Kamanna, V.S., and Kashyap, M.L. 2004. Niacin therapy in atherosclerosis. Curr. Opin. Lipidol. 15:659-665.

95. Tunaru, S., et al. 2003. PUMA-G and HM74 are receptors for nicotinic acid and mediate its antilipolytic effect. Nat. Med. 9:352-355.

96. Cuchel, M., and Rader, D.J. 2006. Macrophage reverse cholesterol transport: key to the regression of atherosclerosis? Circulation. 113:2548-2555.

97. Van Eck, M., et al. 2006. Macrophage ATP-binding cassette transporter $\mathrm{A} 1$ overexpression inhibits atherosclerotic lesion progression in low-density lipoprotein receptor knockout mice. Arterioscler. Thromb. Vasc. Biol. 26:929-934.

98. Repa, J.J., and Mangelsdorf, D.J. 2002. The liver X receptor gene team: potential new players in atherosclerosis. Nat. Med. 8:1243-1248.

99. Wang, N., Lan, D., Chen, W., Matsuura, F., and Tall, A.R. 2004. ATP-binding cassette transporters G1 and G4 mediate cellular cholesterol efflux to highdensity lipoproteins. Proc. Natl. Acad. Sci. U. S. A. 101:9774-9779.

100.Kennedy, M.A., et al. 2005. ABCG1 has a critical role in mediating cholesterol efflux to HDL and preventing cellular lipid accumulation. Cell Metab. 1:121-131.

101.Wang, N., Ranalletta, M., Matsuura, F., Peng, F., 
and Tall, A.R. 2006. LXR-induced redistribution of ABCG1 to plasma membrane in macrophages enhances cholesterol mass efflux to HDL. Arterioscler. Thromb. Vasc. Biol. 26:1310-1316.

102. Ranalletta, M., et al. 2006. Decreased atherosclerosis in low-density lipoprotein receptor knockout mice transplanted with Abcg1-/- bone marrow. Arterioscler. Thromb. Vasc. Biol. 26:2308-2315.

103. Baldan, A., et al. 2006. Impaired development of atherosclerosis in hyperlipidemic Ldlr-/- and ApoE-/mice transplanted with Abcg1-/- bone marrow. Arterioscler. Thromb. Vasc. Biol. 26:2301-2307.

104.Naik, S.U., et al. 2006. Pharmacological activation of liver X receptors promotes reverse cholesterol transport in vivo. Circulation. 113:90-97.

105.Terasaka, N., et al. 2003. T-0901317, a synthetic liver $\mathrm{X}$ receptor ligand, inhibits development of atherosclerosis in LDL receptor-deficient mice. FEBS Lett. 536:6-11.

106.Joseph, S.B., et al. 2002. Synthetic LXR ligand inhibits the development of atherosclerosis in mice. Proc. Natl. Acad. Sci. U. S. A. 99:7604-7609.

107. Levin, N., et al. 2005. Macrophage liver X receptor is required for antiatherogenic activity of LXR agonists. Arterioscler. Thromb. Vasc. Biol. 25:135-142.

108.Li, A.C., and Glass, C.K. 2004. PPAR- and LXRdependent pathways controlling lipid metabolism and the development of atherosclerosis. J. Lipid Res. 45:2161-2173.

109.Groot, P.H., et al. 2005. Synthetic LXR ago- nists increase LDL in CETP species. J. Lipid Res. 46:2182-2191.

110. Chawla, A., et al. 2001. A PPAR gamma-LXR-ABCA1 pathway in macrophages is involved in cholesterol efflux and atherogenesis. Mol. Cell. 7:161-171.

111. Chinetti, G., et al. 2001. PPAR-alpha and PPARgamma activators induce cholesterol removal from human macrophage foam cells through stimulation of the ABCA1 pathway. Nat. Med. 7:53-58.

112.Li, A.C., et al. 2004. Differential inhibition of macrophage foam-cell formation and atherosclerosis in mice by PPAR $\alpha, \beta / \delta$, and $\gamma$.J. Clin. Invest. 114:1564-1576. doi:10.1172/JCI200418730.

113. Oliver, W.R., Jr., et al. 2001. A selective peroxisome proliferator-activated receptor delta agonist promotes reverse cholesterol transport. Proc. Natl. Acad. Sci. U. S. A. 98:5306-5311.

114. Rothblat, G.H., et al. 1999. Cell cholesterol efflux: integration of old and new observations provides new insights. J. Lipid Res. 40:781-796.

115.Zhang, W., et al. 2003. Inactivation of macrophage scavenger receptor class B type I promotes atherosclerotic lesion development in apolipoprotein Edeficient mice. Circulation. 108:2258-2263.

116.Nanje, M.N., et al. 1996. Effects of intravenous infusion of lipid-free apo A-I in humans. Arterioscler. Thromb. Vasc. Biol. 16:1203-1214.

117.Eriksson, M., Carlson, L.A., Miettinen, T.A., and Angelin, B. 1999. Stimulation of fecal steroid excretion after infusion of recombinant proapolipopro- tein A-I: potential reverse cholesterol transport in humans. Circulation. 100:594-598.

118.Nissen, S.E., et al. 2003. Effect of recombinant ApoA-I Milano on coronary atherosclerosis in patients with acute coronary syndromes: a randomized controlled trial. JAMA. 290:2292-2300.

119.Rader, D.J. 2003. High-density lipoproteins as an emerging therapeutic target for atherosclerosis. JAMA. 290:2322-2324.

120. Brewer, H.B., Jr. 2004. Focus on high density lipoproteins in reducing cardiovascular risk. Am. HeartJ. 148(Suppl.):S14-S18.

121.Navab, M., Anantharamaiah, G.M., Reddy, S.T., and Fogelman, A.M. 2006. Apolipoprotein A-I mimetic peptides and their role in atherosclerosis prevention. Nat. Clin. Pract. Cardiovasc. Med. 3:540-547.

122. Garber, D.W., et al. 2001. A new synthetic class A amphipathic peptide analogue protects mice from diet-induced atherosclerosis. J. Lipid Res. 42:545-552.

123. Navab, M. et al. 2002. Oral administration of an Apo A-I mimetic peptide synthesized from D-amino acids dramatically reduces atherosclerosis in mice independent of plasma cholesterol. Circulation. 105:290-292.

124. Navab, M., et al. 2004. Oral D-4F causes formation of pre-beta high-density lipoprotein and improves high-density lipoprotein-mediated cholesterol efflux and reverse cholesterol transport from macrophages in apolipoprotein E-null mice. Circulation. 109:3215-3220 